\title{
A Cost Efficient Real-Time Vehicle Tracking System
}

\author{
Rohit Minni \\ VIT University, Vellore, India \\ 304, Satellite, Om Nagar, \\ Andheri(E), Mumbai-99, India
}

\begin{abstract}
The transportation system plays a vital role in today's world. With the rapid growth in the number of vehicles, the traffic overhead is increasing day by day. People always look for ways to save time while travelling. Due to lack of necessary infrastructure it is difficult to obtain real-time access to a vehicle's location and its timings in case of public bus transportation systems. Addressing this problem, the paper present an innovative methodology to provide real-time updates of the vehicle's location to users through the use of cost efficient smartphones equipped with Global Positioning System that are widely available in the market. The implementation of the system is shown for use in various public and private sectors like for real-time bus tracking, taxi tracking, tracking people, tracking fleet of vehicles for logistics and other companies. The paper also presents how the data collected can be used for traffic estimation and other applications of implementing the system in vehicles.
\end{abstract}

\section{General Terms}

Vehicle Tracking, GPS Positioning, GSM, Applications of Vehicle Tracking.

\section{Keywords}

Cost Efficient; Vehicle Tracking; GPS; Smartphones.

\section{INTRODUCTION}

There has been a rapid growth in the smartphone industry recently. This growth has been consistent with the Moore's Law. The smartphones in the market are available at very reasonable prices and are equipped with functionalities like media players, digital cameras, GPS units, high resolution and sensitive touch-screens, Wi-Fi, internet connectivity and much more. To get the location of a device along with other vital information, the GPS communicates with the satellites (24 operational and 3 extra in case if any fails) ${ }^{[1]}$ whereas, the Global System for Mobile Communications (GSM) relies on the concept of triangulation to acquire the location of a device. The driving factor for this system to function is that at any given time the smartphone will be wirelessly connected to one of the closest base stations ${ }^{[2]}$. The use of internet in smartphones and the amount of data transferred everyday through it is increasing exponentially. This has been made possible by the launch of the $3 \mathrm{G}$ and $4 \mathrm{G}$ services. Despite of all these features provided, these smartphones are available at very affordable prices costing approximately $\$ 75$ to $\$ 150^{[3]}$.

A large portion of the general public today relies on the public means of transport. Most people prefer using the public bus transport system. But, they waste a huge amount of their precious time while waiting at the bus stop for the bus to arrive. Most of the times people miss the buses by a very small margin and cannot decide whether to wait for the next bus or to plan an alternative means for travel. Addressing this critical need of the public, this paper presents an innovative system that tracks the buses in real-time, provides real-time updates about the bus location to the users, gives an estimated arrival time of the bus to the user's current position and maps both the user and the bus on a map.

\section{SURVEY REPORT}

In order to substantiate the viability of the system, an online survey was conducted ${ }^{[4]}$. A questionnaire was created using Google Docs and circulated online by means of social networking websites like Facebook and Google+ and through e-mails. The main intention of the survey was to know the user perspective of the current bus transportation system in India, its drawbacks, the level of dependency on the system, to study the requirements of the frequent travelers and to implement the same in the system. The survey was conducted between 30-Aug-2012 and 06-Sep-2012 and 366 people from India participated in the same.

\subsection{Survey Findings}

Following points can be considered important based on the data collected from the survey. $63 \%$ of the people belonged to the age group of $15-25$ years, $23 \%$ belonged to $25-40$ years, $10 \%$ of them were above 40 years and $3 \%$ of them were below 15 years as depicted in Figure 1 .

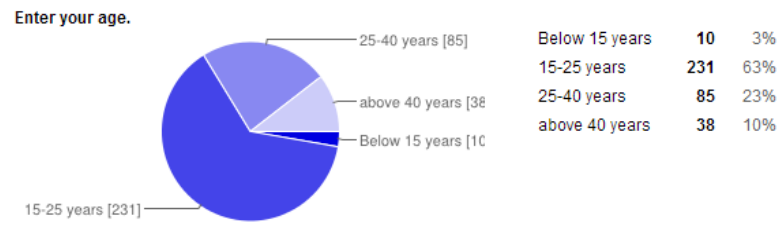

Figure 1. Age Group

$49 \%$ of the people were students, $31 \%$ belonged to the working class and $16 \%$ belonged to the business class as depicted in Figure 2.

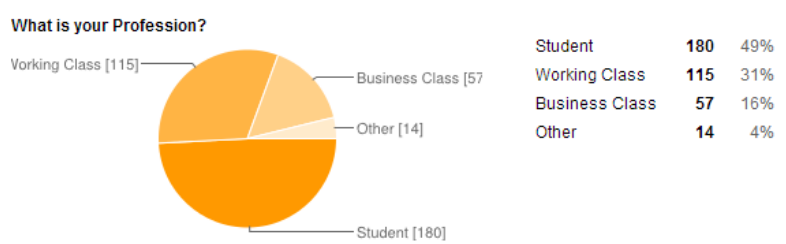

Figure 2 . Profession

When asked about the most preferred means of transport $31 \%$ of the people preferred travelling by train followed by $24 \%$ of the people who preferred travelling by bus as depicted in Figure 3. 

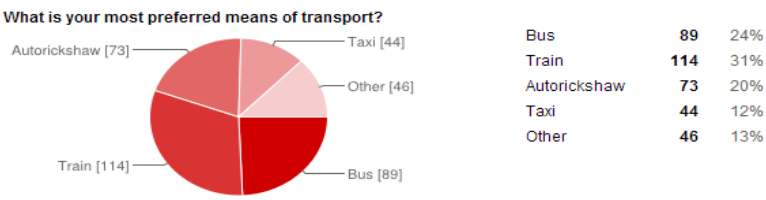

Figure 3. Most Preferred Means of Transport

$10 \%$ of the people said that bus was their only means of transport, 58\% travelled by bus frequently or occasionally and $32 \%$ of them rarely travelled by bus as shown in Figure 4.

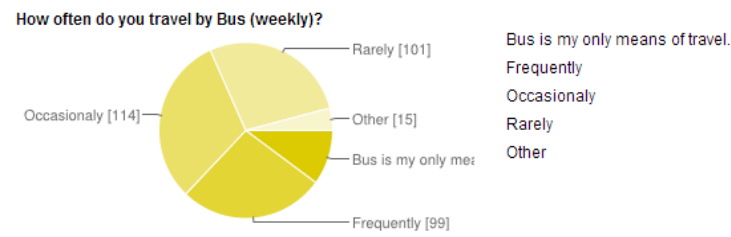

Figure 4. How Frequently People Travel by Bus

A huge percentage i.e., $85 \%$ of the people said that they think that travelling by bus would make them reach late to their destination as illustrated in Figure 5.

Does travelling in Bus Transport gives you a picture of getting late?
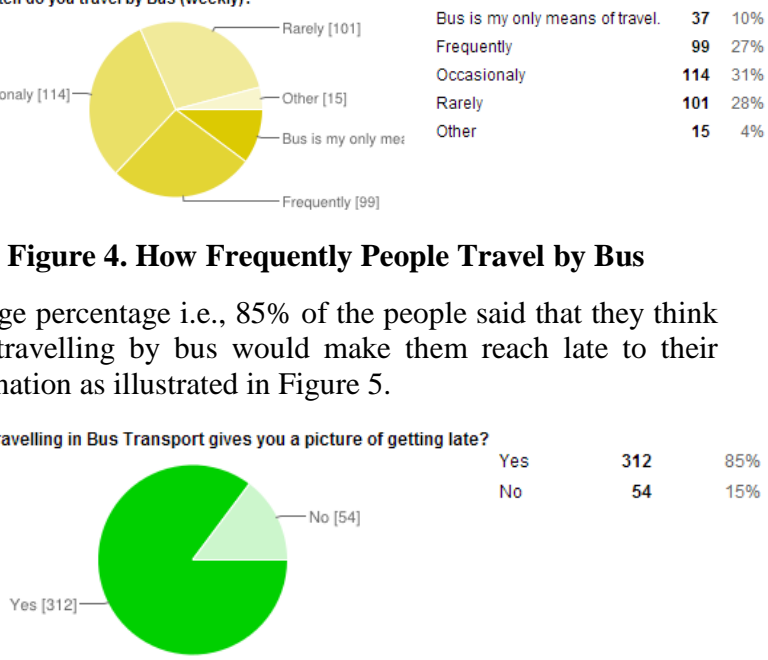

Figure 5. Whether Traveling by Bus gives User the Picture of Getting Late

It is interesting to know that $67 \%$ of the people planned their schedule on the basis of the bus timings and not according to their own convenience as shown in Figure 6.

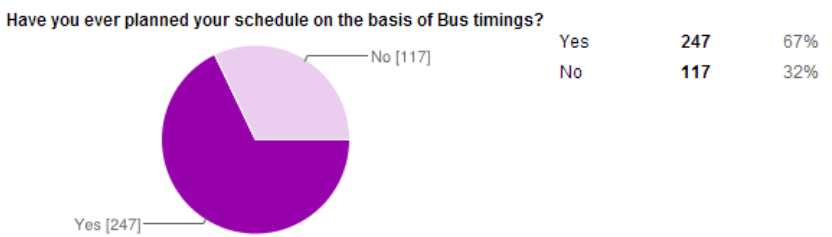

Figure 6. Planning Schedule based on Bus Timings

$84 \%$ of the people missed their bus by a small margin at some point of time as shown in Figure 7.

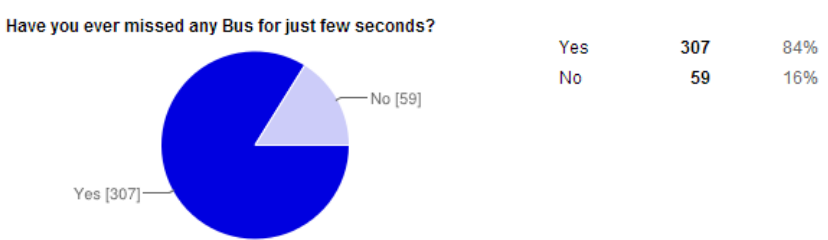

Figure 7. Missed the Bus by a Small Margin

When asked about the average amount of time wasted every day at the bus stop, $22 \%$ of the people said they spent less than 10 minutes, $45 \%$ of them spent $10-20$ minutes, $23 \%$ of them spent $20-30$ minutes and $4 \%$ of them spent more than 30 minutes as depicted in Figure 8.

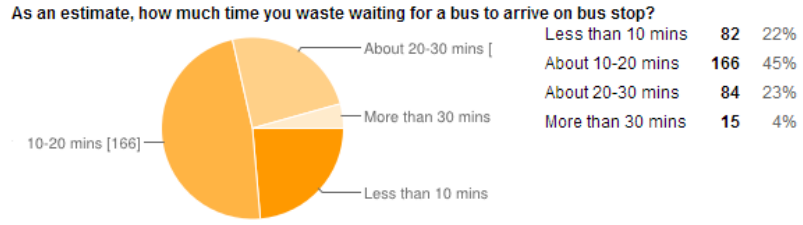

Figure 8. Time Wasted at the Bus Stop

Most of the people spent that time at the bus stop in talking over the phone, gossiping with friends and doing nothing as shown in Figure 9.

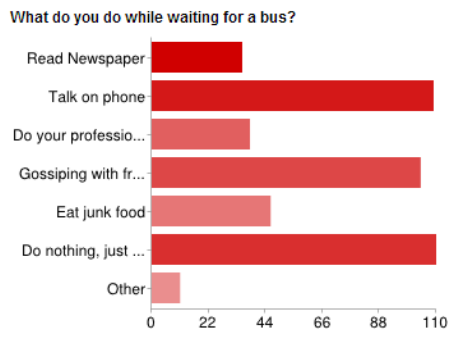

(a) Talk on phone

Do your professional work

Gossiping with friends

Eat junk food

Do nothing, just kill time and wait for bus 110

Other

People may select more than one checkbox, so percentages

Figure 9. Activities Done While Waiting at the Bus Stop

$83 \%$ people believe that the reliability on the bus transportation has increased over the past few years as depicted by Figure 10

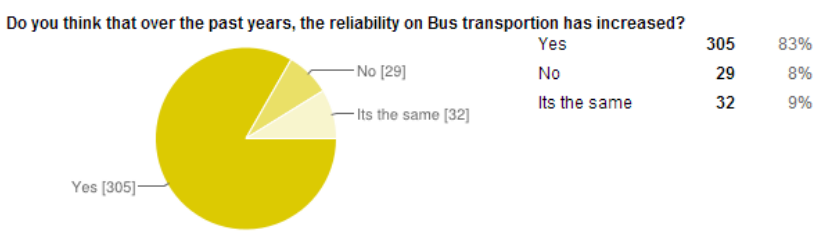

Figure 10. Reliability on Bus Transport System

A high $97 \%$ believe that the bus transportation system in India needs a reform as shown in Figure 11.

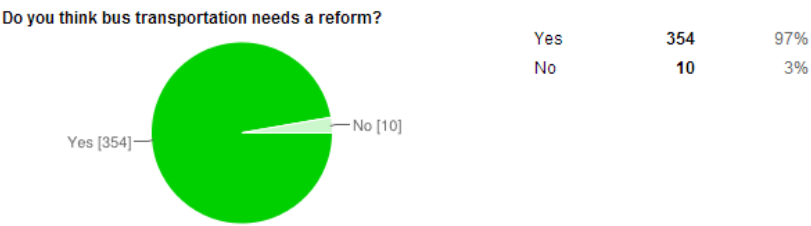

Figure 11. Need for Reformation in Bus Transportation System

\subsection{Survey Analysis}

A huge percentage of the population i.e. $97 \%$ of the people believed that the bus transportation system needed a reform. $24 \%$ of the people preferred traveling by bus. Most of the governments throughout the world promote the public bus transport systems in order to reduce the traffic congestion and to reduce emission of harmful gases by other vehicles to promote sustainable development. It is clearly evident from the survey that the remaining $86 \%$ people do not like traveling by bus because they feel they might reach late to their destination. Generalizing the survey results, on an average a working individual travels daily for $15 \mathrm{~km}$. If these $15 \mathrm{~km}$ were to be travelled in an auto-rickshaw the person would spend an average of Rs. 103 (INR) everyday which is approximately $\$ 2$. Whereas if the same distance is travelled using a bus it would cost approximately Rs. 30 (INR) which is around \$0.6. The Indian workforce comprises of over 400 million people out of which about 96 million travel by bus 
daily. So, if each individual resorts to travel by bus, one can save Rs. 70 (INR) i.e. $\$ 1.4$ daily. This sums up to approximately Rs 6.7 billion of savings each day i.e. about $\$ 0.13$ billion (USD). Thus introducing an effective and reliable bus transportation system is essential considering the given scenario where people refrain from using these services due to various reasons. Most people complained missing the bus by a small margin, they spent over 20 minutes waiting at the bus stop and suggested that it would be beneficial if they received real time updates to the bus locations. The vehicle tracking system that is developed addresses all these issues and makes use of commonly available smartphones that are already owned by most of the individuals. This reduces a major share of the investment cost to setup the system.

The system that is proposed addresses an issue that is faced globally. The system would provide the public with real-time location updates of the buses that they intend to board. It also gives them a list of all the instances of the buses currently running, the distance of the bus from the user, and the estimated arrival time of the bus to where the user is. The user can see the bus on a map too. In this way the user can save a lot of time daily. It also makes the public bus transport system more efficient and reliable. It will definitely make the current system more advanced and would lead to an increase in the number of people preferring buses for their means of transport. The use of buses is spread worldwide and it is essential that its standard be raised so that it becomes the most efficient and fastest means of transportation for one and all.

\section{EXISTING SYSTEM}

The NextBus ${ }^{[5]}$ service in USA uses GPS data combined with advanced prediction and computer modelling to track the buses. It provides details about which bus is going to arrive and when it will arrive using global positioning satellite technology. Each bus is fitted with a GPS Navigator which is very expensive costing $\$ 300$ and upwards.

In Belgium, the STIB Bus service ${ }^{[6]}$ operates that enables tracking through Short Messaging Service (SMS). Waiting times are sent to the customer based on the current position of the vehicle. The system does not provide a graphical and realtime interface to the user.

In London the TFL Bus Service ${ }^{[7]}$ provides real-time information of the buses using bus stop codes, route number, postal codes etc. The information is provided through SMS and live updates on their website.

In India, the BEST Bus Service in Mumbai and the Bangalore Transport Information System tried to provide a tracking system, but the system was very inefficient and erroneous.

The logistics providers and the cab companies do have a tracking system of their own but they again use expensive GPS Navigators and do not provide the tracking information to the common public.

\section{ARCHITECTURAL OVERVIEW AND PROPOSED SYSTEM}

\subsection{Architectural Overview}

The system's main motive is to render a real-time and reliable service to the public facilitating their travel demands. The system makes extensive use of the inbuilt GPS sensor along with its internet capabilities to send and receive data ${ }^{[8]}$. The GPS sensor is used to obtain the current location of the smartphone. If GPS is not available then the location may be obtained using the GSM triangulation method or using the router location from the Wi-Fi connection of the device. The location through GSM triangulation and $\mathrm{Wi}-\mathrm{Fi}$ are automatically managed through the phone's settings and the operating system manages the task of getting the location from the GPS sensor, GSM or Wi-Fi whichever is viable. The GPRS (General Packet Radio Service), 3G or 4G internet connection is used to transmit the data to the web server and to receive the data from the same.

The overview of the system architecture is shown in Figure 12. The architecture provides a unique platform for the integration of various technologies. At the heart of the system lies the Web Server which is deployed on the cloud. The cloud service used for the system deployment is Windows Azure. A mobile service is created on Windows Azure that consists of a SQL Database instance. A table is created that stores the data needed to track the vehicle effectively. This data is then provided to the Bing Maps API to effectively locate the vehicle on a map.

For each transaction of data, two smartphones are needed. The implementation of the App is done on the Windows Phone 8 platform. One phone is with the user while the other is in the vehicle that is to be tracked. The App in the phone in the vehicle updates its location to the database in the cloud along with other details such as the vehicle id, route type etc. A local database in the smartphones is used to store static data such as bus routes, bus numbers, vehicle id, person id, cab/taxi id, etc.

When the App in the user's smartphone queries for the vehicle with the specified vehicle id, the Web Server references the data from the database in the cloud and with the use of Maps API, a list of results is provided to the user in the form of the vehicle's distance from the user. On selecting the required instance of the result, the user is taken to a map in which the exact location of the vehicle and the user is plotted. Along with that, the distance between the two in miles and the estimated arrival time is also displayed.

\subsection{A Test Case for Bus Tracking}

Consider a typical case for a bus where at time $\mathrm{t}$ a bus $\mathrm{X}$ embarks on a journey between $\mathrm{A}$ and $\mathrm{H}$ with intermediate stops $\{\mathrm{B}, \mathrm{C}, \mathrm{D}, \mathrm{E}, \mathrm{F}, \mathrm{G}\}$. Consider another bus $\mathrm{X}^{*}$ which at time $t$ embarks on a journey from $\mathrm{H}$ to $\mathrm{A}$. Two terms are defined here. First the up route which is the route from $\mathrm{A}$ to $\mathrm{H}$ and the other a down route which is from $\mathrm{H}$ to $\mathrm{A}$. This is done since the same bus with the same bus number may travel from $\mathrm{A}$ to $\mathrm{H}$ as well as from $\mathrm{H}$ to $\mathrm{A}$. Hence bus $\mathrm{X}$ follows the up route and the bus $\mathrm{X}^{*}$ follows the down route. Consider an intermediate bus stop C. Say for example the user wishes to go from $\mathrm{C}$ to $\mathrm{H}$. To identify the suitable bus that would take the user from $\mathrm{C}$ to $\mathrm{H}$, an intersection is taken of the buses arriving at bus stop $\mathrm{C}$ and those arriving at bus stop $\mathrm{H}$. It may be represented as $\mathrm{C} \cap \mathrm{H}$ as depicted in Figure 13. Let a user be located at location $\mathrm{Y}$ with location coordinates i.e. latitude and longitude determined by the smartphone. The short listed bus numbers are now queried for their real-time locations and displayed to the user in an ascending order based on the distance between the user and the bus. When the user selects an instance from the results, the user is redirected to a map in which the bus and the user are plotted on the map. The estimated arrival time and distance in miles along with route directions are also provided to the user. The App in the vehicle is made such that it updates the database in the cloud as soon as a change in location is detected. Hence the database is always up to date with the most recent location of the vehicle. 


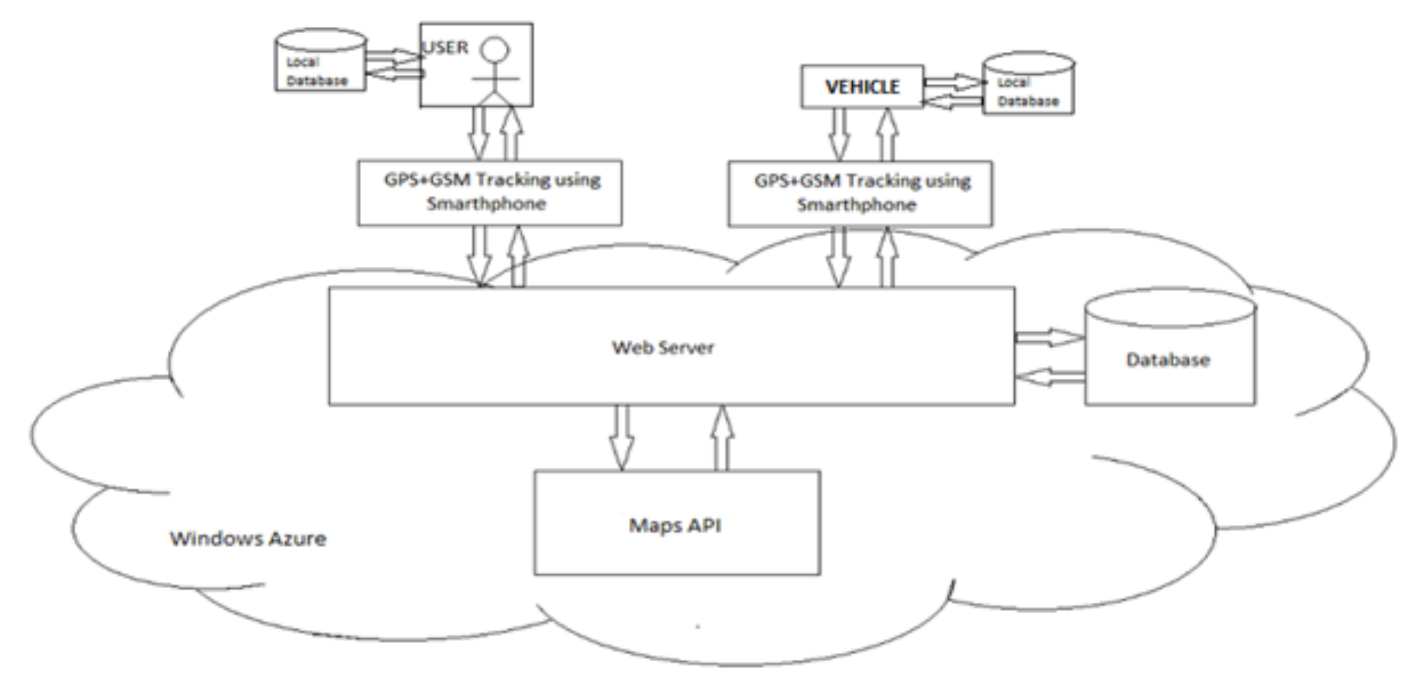

Figure 12. System Architecture

\subsection{Algorithm for the System}

The previously discussed steps can be summarized as follows:

Step1: Get source and destination details from the user or get the desired vehicle id directly from the user.

\section{Step2: Get the location of the user.}

Step3: Get the location of the desired vehicle from the database in the cloud.

Step4: If there are multiple vehicles in the result, calculate the distance for each vehicle from the user and display it in ascending order.

Step5: User selects a particular instance from the result list.

Step6: Display the real-time location of the user and vehicle on a map along with estimated time of arrival, distance in miles and the route directions that the vehicle might follow.

\section{SYSTEM IMPLEMENTATION AND APPLICATIONS}

The base framework for the system has been created. This system can now be modified for specific needs such as bus tracking, taxi or cab tracking, vehicle tracking, tracking people and fleet tracking. There are basically two types of tracking. In the first type, multiple instances of the same ID like in the case of buses is to be tracked and the second type in which a unique ID is tracked. The second type applies to taxi tracking, personal vehicle tracking, tracking people and fleet tracking. A demo of both these types of tracking and how it can be applied to track various types of vehicles like buses, taxis, trucks and even people is presented in this paper.

There are various secondary applications of this system too. For instance, the system can be applied to provide internet to the commuters or people in the vehicle ${ }^{[9]}$. The system can be used to analyze traffic ${ }^{[10]}$ in a particular area and give the information to the user. Since the system makes use of smartphones, it is always connected to the outside world. Quick notifications and alerts can be sent using the smartphones like for example the police or fire brigade can be notified in case of any emergency by means of SMS. Nearby hospitals or ambulance services can also be notified ${ }^{[11]}$ in case of any emergency. Using the accelerometer of the smartphone, the vehicle speed can continuously be monitored and recorded by the system.

\subsection{Bus Tracking}

In case of bus tracking, one smartphone would be installed in the bus. The bus personnel would set the information like the Bus ID, route type (up or down) and enable continuous tracking so that any change in position is recorded in the database. For the given scenario, the Bus ID is taken to be 422 and the bus is currently at the location $(18.9618,72.8228)$. The route type is selected as "Up Route". The continuous location tracking option is enabled so that any change in location results in an update to the database in the cloud. The application interface is shown in Figure 14 and a snapshot of the database in the cloud is provided in Figure 15.

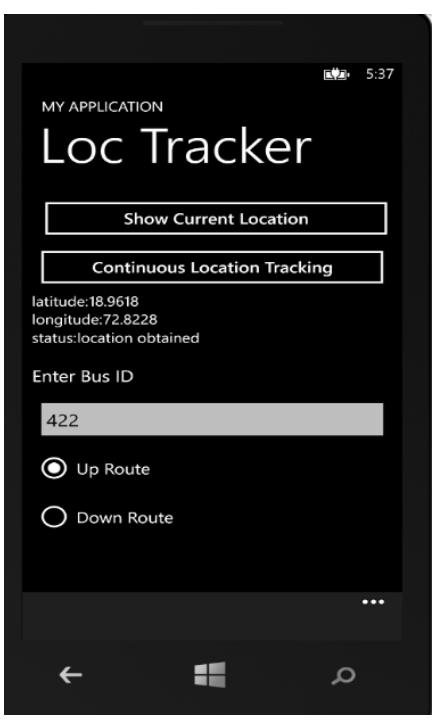

Figure 12. App in the Bus 


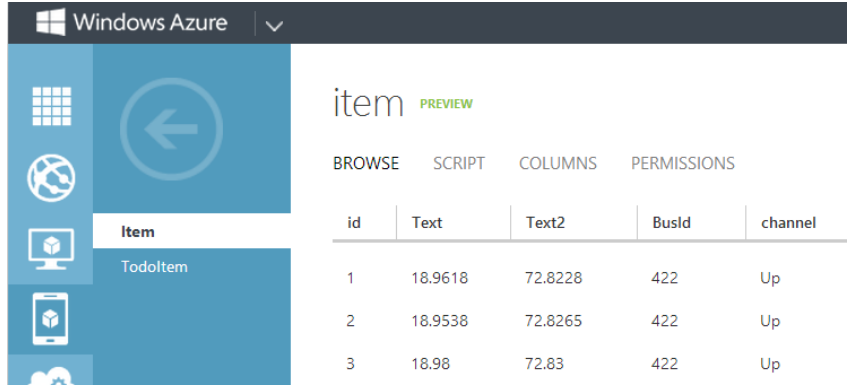

Figure 13. Snapshot of Database Table in Windows Azure

As depicted in Figure 15, there are currently 3 instances of Bus ID 422 running in the Up Route. Now consider that a user
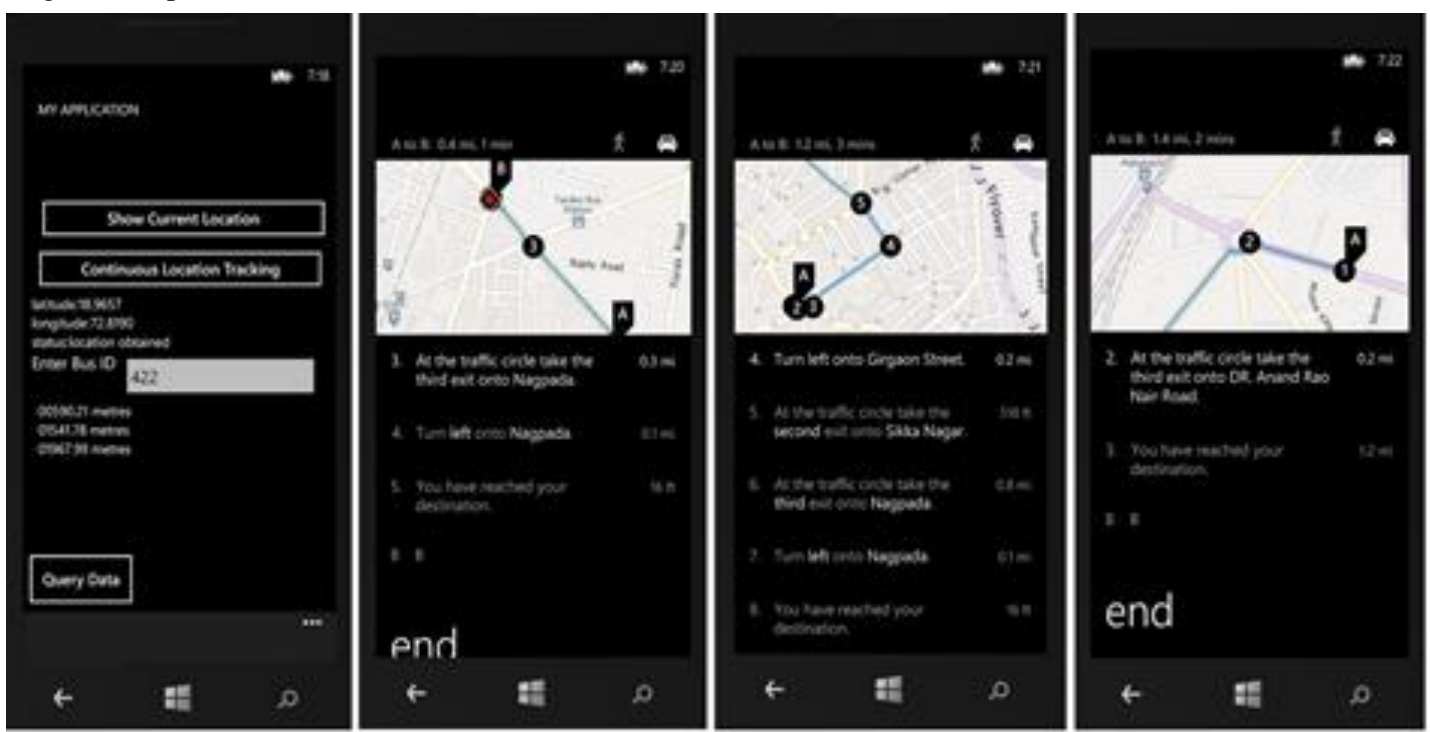

Figure 16. The Application with the User and the 3 Results Returned by the System for a Query to Bus ID 422

\subsection{Taxi or Cab Tracking}

In the case of taxi or cab tracking, a unique Cab ID is used for each instance of cab or taxi. The unique ID will be assigned by the owner of the Cab itself. When a customer books a cab, a unique ID is provided to the customer using which can be used to track the cab before it reaches at the user's location. Also, relatives and friends can monitor one's movements if the customer's unique ID is shared with them. After assigning the unique ID to the customer, the cab driver would set the $\mathrm{Cab}$ ID in the smartphone application in the cab and turn on the continuous tracking option. The application interface for the cab smartphone application is shown in Figure 17.

The snapshot of the database on the cloud is shown in Figure 18. As shown in Figure 17 and Figure 18, there are two cabs running with the Cab ID "21abc" and "22gts". The Cab ID "22gts" is currently at location $(18.98,72.82)$. Now say for example the user has booked the cab with Cab ID " $22 \mathrm{gts".} \mathrm{To}$ locate the cab one has to enter the Cab ID in the mobile application, allow the application to gain location access and press the Query Data button. The distance of the cab from one's current location in meters will be displayed in the app as shown in Figure 19 which in this case is 3456.93 meters. at location $(18.9657,72.8190)$ wishes to query the system for Bus ID 422. The user must enter the desired Bus ID, let the system get the user's current location and then the user can press the query data button. As expected 3 instances of Bus ID 422 are returned by the system in an ascending order of the distance in meters from where the bus is to where the user is currently. Clicking over any of the instance will redirect the user to a map and plot the user and bus location on the map along with the direction list that shows the directions from the bus to the user, estimated time of arrival of the bus and the distance of the bus from the user in miles. The interface for the user application and the results of all the 3 Bus IDs is shown in Figure 16. 


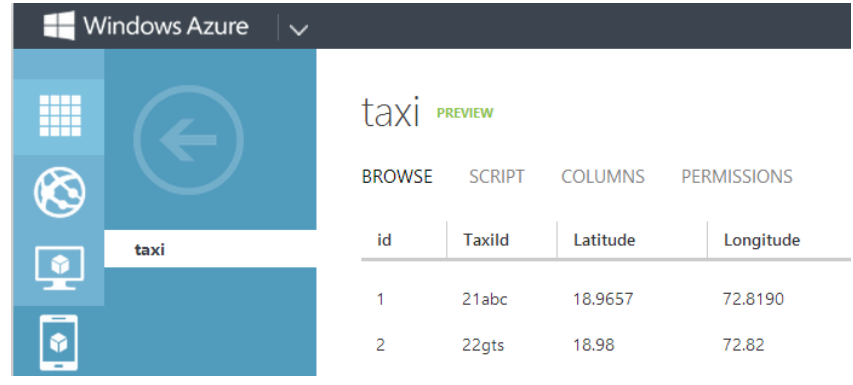

Figure 18. Snapshot of the Database Table

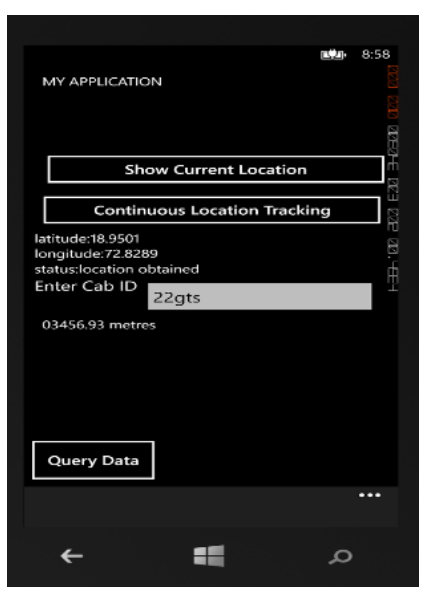

Figure 19. App with the user

When the user clicks on this result, the user would be redirected to the map where the cab and the user would be plotted on a map with the direction list from the cab to the user along with the estimated time of arrival of the cab to the user and the distance of the cab from the user in miles. The result is shown in Figure 20.

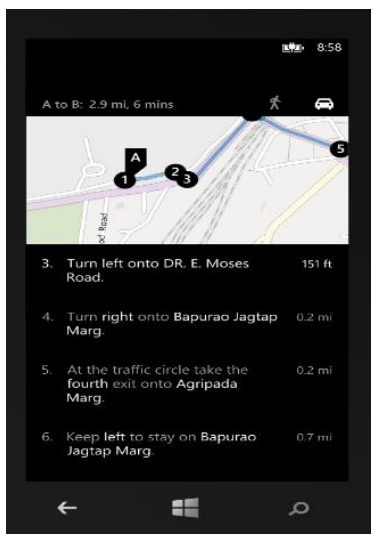

Figure 20. The Result for the Query for Cab ID "22gts"

\subsection{Personal Vehicle Tracking}

The same system that is used to track taxis and cabs can also be used to track personal vehicles. In this case, instead of the Cab ID a unique Vehicle ID is to be used. When a person takes the vehicle out, a unique Vehicle ID is assigned to the smartphone when the continuous tracking option is selected. The real-time location updates would be stored in the database in the cloud. People with whom the unique ID was shared can get to know the current location of the vehicle on the map.

\subsection{Tracking People}

In a similar manner, people can be tracked as well. Each person with the app would be assigned a unique Person ID. When the person goes out and turns on continuous location tracking, the location would be updated in the database in the cloud. People with whom the ID was shared can track the person from time to time.

This particular application is especially useful for women who travel late in the night. They may turn on continuous location tracking whenever they are out especially at night. The family members can continuously monitor the whereabouts of the person with the app. A panic button can also be implemented in the app that would notify a specific person in case of emergency by means of a SMS containing the last known location of the person.

\subsection{Tracking Fleet of Vehicles}

Most of the logistics and other manufacturing companies have a large number of vehicles with them. They transport goods worth millions daily. This system provides them with an economic yet efficient method to track and monitor the movements of their vehicles. The truck driver would set the unique ID and turn on continuous tracking. The truck owner can monitor and track the location of the truck in real-time. Also, if the truck is carrying parcels of customers, the unique ID may be shared with the customers. At present logistics companies provide tracking of parcels, but the data is provided only when the parcel is scanned at a hub. Using this system, the customer can continuously monitor the parcel location thus making the whole process more transparent to the user.

\subsection{Secondary Applications of the System}

\subsubsection{Providing Internet to the Commuters}

Most of the smartphones available today possess Wi-Fi functionality in them. Using the Wi-Fi module a hotspot can be created to which up to eight devices could connect and get access to the internet of the smartphone in which the hotspot is created. But research is still going on and every year the number of devices that can connect is increasing. Having an internet facility for the commuters in a taxi or bus is a great option and will benefit the commuters at large especially if they are tourists. This facility would greatly improve the image and standard of the company thus attracting more customers.

\subsubsection{Providing Traffic Analysis}

Since there are various applications of the system, a lot of data would be accumulated. The speed of the vehicles can be obtained through the use of accelerometers in the smartphone [12]. When a request for traffic update in a particular area is received, the system would cross reference the location coordinates of the requested area with the vehicles in the nearby area. An average of the speed of the vehicles in the nearby area that use this tracking system would be used to determine the traffic status in that area. This method is viable since at any given moment, there are a large number of taxis, buses and vehicles at most of the streets. If the average vehicle speed is around $5-10 \mathrm{kmph}$, it can estimate that the area is crowded with vehicles and the user should opt for an alternative route. If the data is insufficient to estimate the traffic in that area an appropriate message would be given to the user. 


\subsubsection{Emergency Services}

The app can be used to notify the police stations nearby about any crime or suspicious activity. The nearby police stations can be found out using the Maps APIs. A SMS message would be sent to the concerned authorities.

In case of a fire breakout a notification can be sent to nearby fire stations. Similarly, in case of accidents nearby hospitals may be notified for help.

\section{CONCLUSION}

Thus in this paper an economic yet efficient method of tracking vehicles is presented. The development of the system is sufficiently motivated through the collection of the survey results. The system was made so that it incorporated most of the needs of the people as expressed in the survey. The algorithm designed is robust, light-weight and very practical. The system was successfully implemented and tested for various applications such as bus tracking, cab or taxi tracking, personal vehicle tracking, tracking people and tracking fleet of vehicles. Once a smartphone is installed in the vehicle it has a great number of secondary applications. It can be used to provide internet facilities to the commuters through the use of mobile hotspots.

The systems that are currently used for tracking vehicles involve expensive GPS Navigators and complex software. On the other hand, this system is very economical to implement as most of the users already possess smartphones. This system provides fast and accurate updates of the location of the vehicles to the users. By implementing this system in the public buses, the chances that people miss a bus would greatly reduce thus increasing the reliability of the bus transportation systems. A huge amount of money would be saved daily if people traveled by buses and the amount of traffic and pollution would also reduce substantially. Future enhancements of the system would include traffic analysis and emergency notifications and deploying the system on a wide range of mobile platforms like Android and iOS.

\section{ACKNOWLEDGEMENTS}

The participation of the volunteers in the online survey is greatly appreciated for their valuable suggestions and inputs.
Prof. Dr. Krishna Chandramouli and Prof. C. Ranichandra also provided their valuable inputs and feedback on the system's implementation.

\section{REFERENCES}

[1] Imam M. Almomani, Nour Y. Alkhalil, Enas M. Ahmad, Rania M. Jodeh. Ubiquitous GPS Vehicle Tracking and Management System. In Proc. IEEE Jordan Conference on AEECT 2011, 1-6.

[2] Overview of Location Technologies - Openwave. http://www.ipcgps.com/uploads/docs/Intro_to_Location_ Technologies-1.pdf.

[3] Moloo, R.K. Low-Cost Mobile GPS Tracking Solution. In Proc. BCGIN 2011, 516-519

[4] A Survey on the Current Bus Transportation System https://docs.google.com/spreadsheet/viewform?formkey= dDVVYmY2T2VUNGJjVmg4clRjVlB3b0E6MQ.

[5] How NextBus Works. http://news.nextbus.com/how-nextbus-works-2/.

[6] STIB Real Time Mobile Information. http://www.stib.be/realtime_gsm.html?l=en

[7] TFL Live Bus Arrival Information. http://countdown.tfl.gov.uk/\#/

[8] Manoharan, S. On GPS Tracking of Mobile Devices. In Proc. ICNS 2009, 415-418

[9] Keshav K., Indukuri V.R., Venkataram P. Energy Efficient Scheduling in 4G Smart Phones for Mobile Hotspot Application. In Proc. NCC 2012, 1-5.

[10] Menard T., Miller J. FreeSim_Mobile: A Novel Approach to Real-Time Traffic Gathering using the Apple iPhone ${ }^{\mathrm{TM}}$. In Proc. VNC 2010, 57-63.

[11] Suganthi J., Umareddy N.V., Awasthi N. Medical Alert Systems with TeleHealth and TeleMedicine Monitoring using GPS and GSM Technology. In Proc. ICCCNT 2012, 1-5.

[12] Li-jun Wu. Experimental Study on Vehicle Speed Estimation using Accelerometer and Wheel Speed Measurements. In Proc. MACE 2011, 294-297. 